\title{
The adjusted clinical group (ACG) is adapted to predict costs of chronic disease N Upakdee
}

\author{
Address: Department of Pharmacy Practice, Faculty of Pharmaceutical Sciences, Naresuan University, Phitsanulok, Thailand \\ from 25th Patient Classification Systems International (PCSI) Working Conference \\ Fukuoka, Japan. II-I4 November 2009 \\ Published: 5 November 2009 \\ BMC Health Services Research 2009, 9(Suppl I):AI4 doi:I0.II86/I472-6963-9-SI-AI4
}

This abstract is available from: http://www.biomedcentral.com/|472-6963/9/SI/AI4

(c) 2009 Upakdee; licensee BioMed Central Ltd.

\section{Objective}

(1) To determine chronic illness costs for outpatients at the provincial hospital, (2) to develop a prospective model predicting total costs using demographic and clinical information including the Adjusted Clinical Group (ACG) and Charlson Comorbidity Index (CCI).

\section{Methods}

A retrospective study included 2,433,027 patients attending 22 large general and provincial hospitals. Data, including diagnostic and resource utilization, were obtained over the one-year period of 2008. Hospital and pharmacy cost data for outpatients were obtained from a hospital-based computer system. The multiple linear regression technique was used for constructing the prediction model. The dependent variable was the natural logarithm of reimbursed money. The output that would result from replacing the ACG with the CCI variable was considered, and the two possible outputs were compared.

\section{Results}

Average annual per patient cost was THB 1,489. Pharmacy costs were THB 343 , accounting for $23 \%$ of the total outpatient costs. In the predictive model, statistically significant predictors were composed of sex, age, health insurance scheme, chronic diseases (such as diabetes, hypertension, asthma, chronic renal failure, etc.), and the ACG or CCI. When the CCI was replaced by the ACG, using the number of aggregated diagnostic groups (ADG) and major $\mathrm{ADG}$, the adjusted $\mathrm{R}^{2}$ changed from 0.249 to.0.301.

\section{Conclusion}

The comorbidity index adapted from ACG had a higher influence on the predictive model than the CCI. In an outpatient setting, a simple count of diagnoses may be the most efficient comorbidity measure for predicting utilization and healthcare costs over the year. 